\title{
Analyse der Wirkmechanismen im fluidfreien Wälzkontakt mit beschichteten Oberflächen
}

\author{
Sebastian Sklenak ${ }^{1}$ (D) - Jens Brimmers ${ }^{1} \cdot$ Christian Brecher $^{1}$ \\ Eingegangen: 26. März 2021 / Angenommen: 30. Juli 2021 / Online publiziert: 13. September 2021 \\ (c) Der/die Autor(en) 2021
}

\section{Zusammenfassung}

Der Zahnkontakt wird in Zahnradgetrieben in der Regel mit flüssigem Schmierstoff, wie beispielsweise Öl oder Fett geschmiert. Jedoch führen extreme Betriebsbedingungen, wie beispielsweise hohe Druck- und Temperaturschwankungen, bei flüssigen Schmierstoffen zum Ausfall der positiven Schmiereigenschaften. Für den Einsatz von Zahnradgetrieben unter fluidfreien Betriebsbedingungen bieten Festschmierstoffe eine alternative Schmierung im Wälzkontakt. Das Ziel dieser Arbeit ist die Kenntnis über die Wirkmechanismen im PVD-beschichteten (Physical Vapour Deposition - Physikalische Gasphasenabscheidung) und fluidfreien Wälzkontakt. Für den fluidfreien Einsatz im Zahnkontakt wurde ein Schichtsystem auf Basis von Molybdändisulfid im Zahnrad-Analogieversuch auf dem Zwei-Scheiben-Reibkrafttribometer untersucht. Anhand der Charakteristik der Reibkraft kann zwischen einer Gebrauchs- und Lebensdauer unterschieden werden. Dabei zeigt die Lebensdauer eine bessere Wiederholgenauigkeit als die Gebrauchsdauer. Für die Kenntnisse über die Verschleißmechanismen wurden in Bezug auf Gebrauchsdauer und Lebensdauer Versuche mit Intervallbetrieb durchgeführt und ausgewertet. Die Verschleißanalyse der Kontaktfläche auf der Prüfwelle zeigt, dass sich bereits zu Beginn einer instationären Reibungsphase im Prüflauf ein starker Verschleiß in einem großen Teil der Laufbahn entwickelt hat.

\section{Analysis of the mechanisms of action within the dry lubricated rolling-sliding contact of coated surfaces}

\begin{abstract}
Tooth contacts in gear drives are usually lubricated with liquid lubricants, such as oil or grease. However, extreme operating conditions with high pressure and temperature fluctuations cause liquid lubricants to lose their positive lubricating properties. For the use of dry lubricated and mechanically highly loaded gearing, the operation of different $\mathrm{MoS}_{2}$ coatings in tooth contact is the subject of current research in the Priority Program 2074 of the DFG (german research foundation). The aim of this work is to gain knowledge about the mechanisms of action in PVD-coated (physical vapour deposition) and fluid-free rolling-sliding contact. For fluid-free use in tooth contact, a coating system based on molybdenum disulfide was investigated in a gear analogy test on the disk-on-disk friction tribometer. Based on the characteristics of the behavior of the frictional force, a distinction can be made between life duration and use duration. Here, the life duration shows better repeatability than the use duration. For knowledge of the wear mechanisms, tests with interval operation were carried out and evaluated in relation to life duration and use duration. The wear analysis of the contact surface on the test shaft shows that heavy wear has already developed in a large part of the raceway at the start of a transient phase in the test run.
\end{abstract}

Sebastian Sklenak

s.sklenak@wzl.rwth-aachen.de

Jens Brimmers

j.brimmers@wzl.rwth-aachen.de

Christian Brecher

c.brecher@wzl.rwth-aachen.de

1 Werkzeugmaschinenlabor, RWTH Aachen, Campus-Boulevard 30, 52074 Aachen, Deutschland

\section{Einleitung und Motivation}

Bei Zahnradgetrieben wird der Wälzkontakt an den Zahnflanken üblicherweise mit flüssigem Schmiermittel, wie Öl oder Fett, geschmiert. Dabei wird durch den flüssigen Schmierstoff eine Trennung der mechanisch hochbelasteten Zahnflanken angestrebt [1,2]. Durch die Kombination von flüssigem Schmierstoff und der Kinematik des Wälzkontakts baut sich im Zahnkontakt ein elastohydrodynamischer 
Abb. 1 Verschleiß im fluidfreien Zahnkontakt
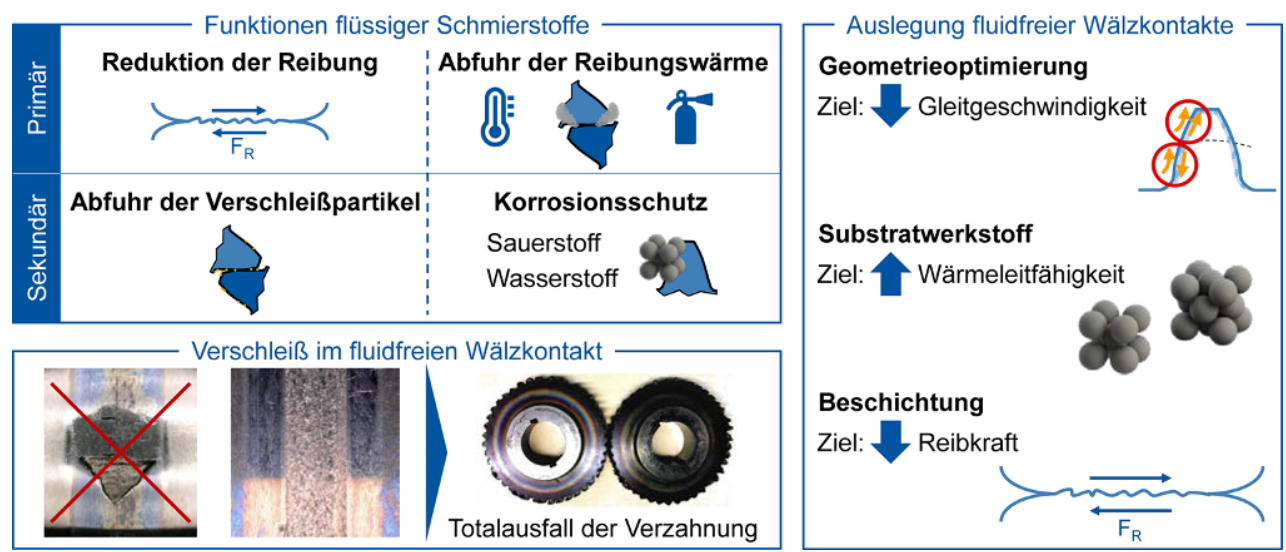

Schmierfilm auf. Die primären Funktionen dieses Schmierfilms sind die Reduzierung der Reibung und die Abfuhr der Reibenergie, Abb. 1. Des Weiteren dient der flüssige Schmierstoff zur Abfuhr von Verschleißpartikeln und zum Schutz vor Korrosion der Zahnräder. Der Einsatz flüssiger Schmierstoffe in Zahnradgetrieben ist jedoch aufgrund der Schmierstoffeigenschaften begrenzt [3]. Bei Ölen und Fetten können extreme Druck- und Temperaturschwankungen, wie beispielsweise in der Luft- und Raumfahrt, zu einer Ausgasung führen, sodass der Schmierstoff seine reibungsreduzierende Wirkung verliert. Neben extremen Einsatzbedingungen können auch Hygienevorschriften, wie beispielsweise in der Lebensmittelindustrie den Einsatz von flüssigen Schmierstoffen begrenzen [4].

Untersuchungen bei mangelhafter Schmierstoffversorgung im Zahnradgetriebe zeigen, dass sich die Lebensdauer einer konventionell ausgelegten Verzahnung deutlich reduziert und die Unterbrechung der Schmierstoffversorgung frühzeitig zum Totalausfall des Getriebes führt [5]. Des Weiteren weicht die Schadensart gegenüber dem Ermüdungsschaden im flüssiggeschmierten Wälzkontakt ab. Die in Abb. 1 dargestellte Verzahnung zeigt einen Schaden infolge unterbrochener Schmierstoffversorgung. Die Verfärbungen an den Stirnseiten der Zahnräder weisen auf den Einfluss hoher Temperaturen im Zahnkontakt hin. Für eine fluidfreie Anwendung von Zahnradgetrieben ist somit eine gezielte Auslegung des Zahnkontakts notwendig.

Für den Einsatz fluidfreier Zahnradstufen werden im Allgemeinen drei Ansätze zur Auslegung der Verzahnung unterschieden, siehe Abb. 1. Ein Ansatz fokussiert die Reduzierung der Gleitgeschwindigkeit im Zahnflankenkontakt durch eine optimierte Verzahnungsgeometrie [6, 7]. Mit der Reduzierung der Gleitgeschwindigkeit im Wälzkontakt kann die thermische Beanspruchung und der Verschleiß im Zahnkontakt reduziert werden.

Ein weiterer Ansatz für die Reduzierung der thermischen Beanspruchung im Wälzkontakt ist die Wahl eines Grundwerkstoffs mit einer hohen Temperaturbeständigkeit und guten Wärmeleitfähigkeit. Dabei kommen neben hochle- gierten Stählen und Aluminium- und Titanlegierungen auch Kunststoffe für fluidfreie Zahnradstufen zum Einsatz [3, 8, 9]. Keramische Werkstoffe zeigen bei fluidfreien Anwendungen in Wälzlagern hohe thermische Beständigkeit und eine anwendungsnahe Lebensdauer. Aufgrund der stoßartigen Spannungsspitzen eignen sich keramische Werkstoffe für den mechanisch hochbelasteten Zahnkontakt jedoch nicht.

Der dritte Ansatz zur Auslegung eines fluidfreien Zahnkontakts basiert auf der Reduzierung der Reibung mithilfe von Festschmierstoffen. Kunststoffbasierte Festschmierstoffe, wie beispielsweise Polytetrafluorethylen (PTFE), weisen bei einer Relativbewegung im Kontakt einen sehr niedrigen Reibkoeffizienten auf. Jedoch hat PTFE gegenüber anderen Festschmierstoffen einen relativ eng begrenzten thermischen Einsatzbereich [10]. Neben kunststoffbasierten Festschmierstoffen kommen auch Weichmetalle wie Gold, Silber und Kupfer, zum Einsatz. Dabei zeigen Weichmetalle im Wälzkontakt von Wälzlagern eine bessere thermische Beständigkeit gegenüber PTFE. Einen weiteren Ansatz für einen fluidfreien Betrieb von Zahnradstufen bieten Festschmierstoffe in Form von Schichtgitterwerkstoffen, wie beispielsweise Graphit und Molybdändisulfid $\left(\mathrm{MoS}_{2}\right)$ [11]. Für fluidfreie Anwendungen kommen bereits lebensdauergeschmierte Wälzlager mit einer $\mathrm{MoS}_{2}$ Beschichtung zum Einsatz [12]. Für den Einsatz einer fluidfreien und mechanisch hochbelasteten Verzahnung ist der Betrieb unterschiedlicher $\mathrm{MoS}_{2}$-Beschichtungen im Zahnkontakt Gegenstand aktueller Forschung im Schwerpunktprogramm 2074 der DFG.

\section{Stand der Technik}

Im Allgemeinen beschreibt der Verschleiß einen fortscheitenden Materialverlust aus den Kontaktflächen der Wälzkörper durch mechanische Ursachen [13]. Die Kenntnis über den Verschleiß in Bezug auf die Lebensdauer ist für eine gezielte Auslegung eines Wälzkontakts entscheidend. 
Abb. 2 Verschleißverhalten im feststoff- und flüssiggeschmierten Wälzkontakt [12]
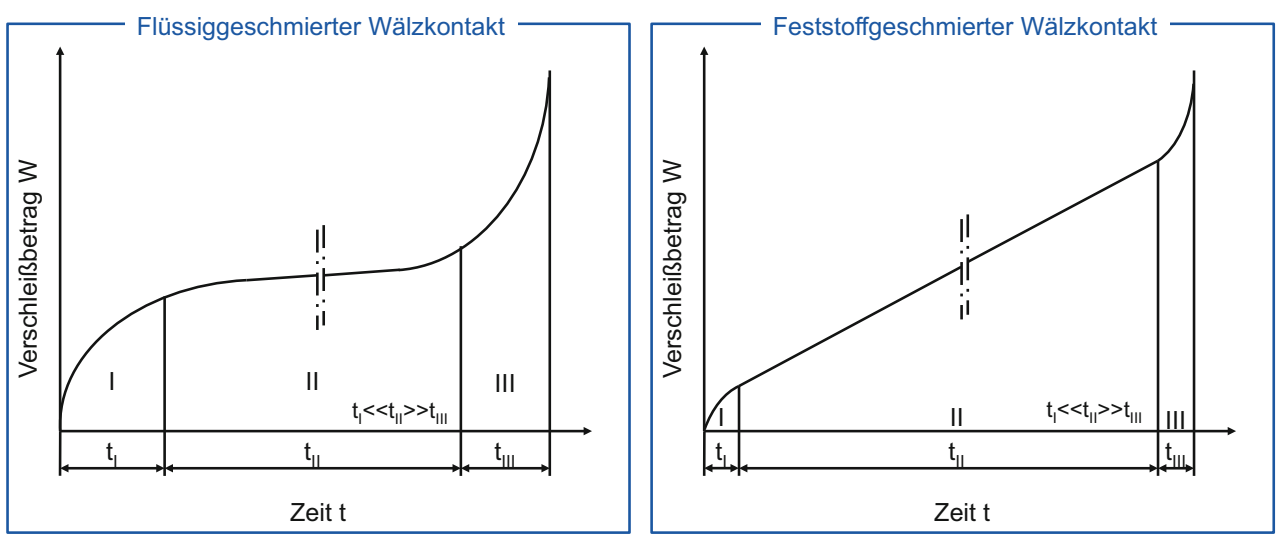

Der Verschleiß kann dazu für flüssiggeschmierte und feststoffgeschmierte Wälzkontakte, wie in Abb. 2 dargestellt, in drei Verschleißphasen unterteilt werden.

Im flüssiggeschmierten Kontakt verändern sich in der ersten Verschleißphase zunächst große Rauheitsspitzen, sodass sich die Oberfläche infolge Deformation einebnet. Dieser Effekt wird als Einlauf bezeichnet. In der zweiten Verschleißphase kommt es nur zu vereinzeltem Festkörperkontakt. Dadurch findet in der zweiten Verschleißphase nahezu kein Materialabtrag statt. In der dritten Verschleißphase entsteht durch Ermüdungserscheinungen zunächst ein erhöhter Materialabtrag. Im weiteren Verlauf führen Verschleißpartikel im Wälzkontakt zu einer deutlichen Zunahme des Materialabtrags [12].

Das zeitabhängige Verschleißverhalten im feststoffgeschmierten Wälzkontakt (siehe Abb. 2) unterscheidet sich grundsätzlich von dem flüssiggeschmierten Wälzkontakt. Im Einlaufvorgang (Verschleißphase I) nimmt der Verschleiß aufgrund der initialen Beanspruchung des Festschmierstoffs zu. Diese Zunahme tritt je nach Kontaktbedingungen beispielsweise durch Transfermechanismen und/oder Umorientierung der Gleitebenen auf. Die zweite Verschleißphase beschreibt einen Zustand mit stationärem Verschleiß. Diese Verschleißphase weist eine weitgehend konstante Verschleißrate auf, bis eine kritische Schichtdicke des Festschmierstoffs unterschritten wird. In der dritten Verschleißphase steigt der Verschleiß stark an. Die Festschmierstoffschicht wird lokal an einzelnen Rauheitsspitzen durchbrochen, sodass der Substratwerkstoff frei liegt. Mit weiteren Lastzyklen kommt es infolge Adhäsion und übermäßigen Verschleiß der Kontaktoberflächen zu einem Totalausfall des Wälzkontakts [12].

Die Lebensdauer einer Verzahnung basiert auf genormten Tragfähigkeitsberechnungen in Bezug auf definierte Verzahnungsschäden. Die Tragfähigkeit einer Verzahnung kann für spezifische Schäden, wie beispielsweise Grübchen, Graufleckigkeit und Fressen, nach ISO 6336 berechnet werden [14]. Aufgrund der abweichenden Verschleißmechanismen zwischen feststoff- und flüssiggeschmierten
Wälzkontakt weichen Schadenform und die Definition der Lebensdauer voneinander ab. Für den feststoffgeschmierten Wälzkontakt wird in der Literatur zwischen einer Gebrauchs- und einer Lebensdauer unterschieden [12]. Das Einsatzverhalten eines feststoffgeschmierten Wälzkontakts mit $\mathrm{MoS}_{2}$-Festschmierstoff kann für stationäre Betriebsbedingungen in zwei Reibungsphasen eingeteilt werden. Die erste Reibungsphase zeichnet sich durch einen nahezu stationären Zustand der Reibkraft aus und wird durch die Gebrauchsdauer begrenzt. In der zweiten Reibungsphase wird bei Versuche im Wälzlager eine instationäre Reibkraft mit einem anschließenden starken Anstieg beobachtet [15].

Molybdändisulfid $\left(\mathrm{MoS}_{2}\right)$ gehört $\mathrm{zu}$ den Schichtgitter-Festschmierstoffen, die sich durch ihren besonderen Schmiermechanismus kennzeichnen. Bei einer tribologischen Beanspruchung führen ausgeprägte Gleitebenen innerhalb der Schichtgitterstruktur zu einer internen Scherung. Bei angemessener Auslegung des Tribokontakts verhindern die molekularen Kräfte zwischen den Gleitebenen ein Abblättern oder Abplatzen einzelner Schichtpartikel [12]. $\mathrm{MoS}_{2}$ zeichnet sich als Festschmierstoff besonders durch einen sehr niedrigen Reibkoeffizienten $\mu<0,1$ aus [16]. Die Verschleißrate von $\mathrm{MoS}_{2}$-Schichten wird von der relativen Luftfeuchtigkeit beeinflusst, da eine erhöhte Luftfeuchtigkeit die Oxidation der Beschichtung begünstigt. Das Verschleißverhalten bei atmosphärischen Bedingungen mit erhöhter Luftfeuchtigkeit kann einerseits durch den Beschichtungsprozess verbessert werden. Andererseits können Fremdatome, wie beispielsweise Titan, im $\mathrm{MoS}_{2}$ Schichtsystem den Einfluss von erhöhter Luftfeuchtigkeit auf das Reibverhalten reduzieren [16-18].

Zusammenfassend folgt aus dem Stand der Technik, dass Festschmierstoffe als Beschichtungen einen Ansatz für die Auslegung eines fluidfreien Zahnkontakts darstellen. Jedoch unterscheidet sich das Einsatzverhalten in Bezug auf Verschleiß und Reibung eines feststoffgeschmierten Wälzkontakts grundlegend von dem eines flüssiggeschmierten Wälzkontakts. Das Reibungs- und Verschleißverhalten wurde für den Punktkontakt, wie beispielsweise für Wälzla- 
Abb. 3 Zielsetzung und Vorgehensweise

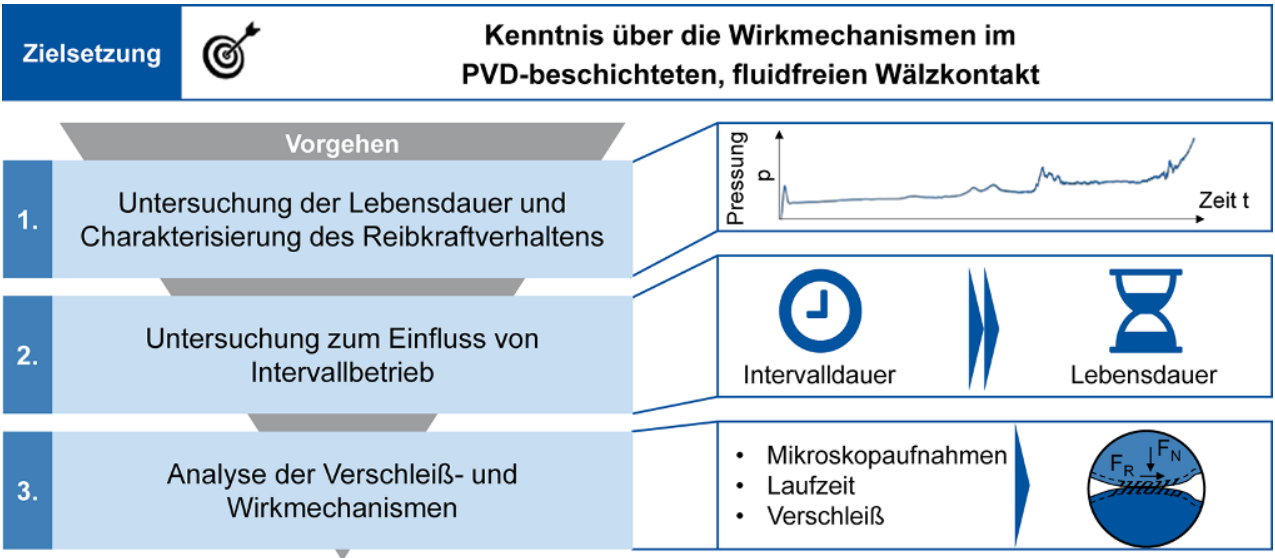

geranwendungen, bereits detailliert untersucht. Für die Anwendung von Festschmierstoffen im Linienkontakt einer Verzahnung unter hoher mechanischer Beanspruchung und ohne flüssige Schmiermittel sind die Verschleißmechanismen noch weitestgehend unbekannt. Insgesamt sind die Verschleißmechanismen und Einflussgrößen im feststoffgeschmierten Wälzkontakt noch nicht in dem Umfang erforscht, wie es für den flüssiggeschmierten Wälzkontakt der Fall ist.

\section{Zielsetzung und Motivation}

Das Ziel dieser Arbeit ist die Erweiterung der Kenntnisse über die Wirkmechanismen im PVD-beschichteten und fluidfreien Wälzkontakt. Die Vorgehensweise stützt sich auf experimentelle Untersuchungen mit einem Zahnrad-Analogieprüfstand. Zur Erreichung des Gesamtziels wird das Vorgehen gemäß Abb. 3 in drei Schritte unterteilt.

Im ersten Schritt wird die Befähigung des Zahnrad-Analogieprüfstands zur Untersuchung und Charakterisierung trockener Wälzkontakte geprüft. In mehreren Versuchsläufen wird die Wiederholbarkeit der Versuchsergebnisse anhand der Lebensdauer quantifiziert. Des Weiteren wird das Reibkraftverhalten im Prüflauf charakterisiert.

Für die gezielte Untersuchung der Wirkmechanismen im feststoffgeschmierten Wälzkontakt ist eine messtechnische Erfassung der Kontaktflächen notwendig. Aus dem Stand der Technik ist bekannt, dass die Temperatur im fluidfreien Wälzkontakt aufgrund unzureichender Kühlung der Wälzkörper ein instationäres Verhalten aufweist. Deshalb wird im zweiten Schritt der Einfluss von Unterbrechungen auf die Lebensdauer im PVD-beschichteten und fluidfreien Wälzkontakt untersucht. Mit Prüfintervallen in unterschiedlichen Reibungsphasen des Prüflaufs wird der Einfluss auf das charakteristische Reibkraftverhalten untersucht.

Die Analyse der gesammelten Messdaten aus den experimentellen Versuchen ermöglicht im dritten Schritt einen detaillierten Einblick in das Verhalten der Beschichtung im fluidfreien Wälzkontakt unter hoher mechanischer Beanspruchung. Auf Basis der Analyse von lichtmikroskopischen Aufnahmen und Profilmessungen werden Rückschlüsse auf Verschleiß- und Transfermechanismen gezogen.

\section{Untersuchungsmethode}

Die primären Herausforderungen bei der experimentellen Untersuchung des Zahnflankenkontakts sind die isolierte Betrachtung einzelner Schadens- bzw. Verschleißmechanismen und den daraus resultierenden vielfältigen Auswirkungen auf das Einsatzverhalten. Für eine fokussierte Untersuchung des Zahnflankenkontakts wird ein definierter Wälzwinkel im Zahnrad-Analogieprüfstand (hier: Zwei-Scheiben-Tribometer, Abb. 4) abgebildet [19, 20].

Der feststoffgeschmierte Wälzkontakt unterscheidet sich sowohl im Einsatzverhalten als auch im Verschleißverhalten grundlegend vom flüssiggeschmierten Wälzkontakt, sodass beispielsweise eine fehlende Abfuhr der Reibungswärme aus dem Kontaktbereich die isolierte Untersuchung spezifischer Einflüsse erschwert. Für die in dieser Arbeit analysierten Versuche wurden jeweils die Prüf- und Gegenwelle mit einem $\mathrm{MoS}_{2}$-Schichtsystem in einem PVD-Verfahren (Beschichtungsanlage CemeCon CC800/9 Custom) am Leibnitz-Institut für Werkstofforientierte Technologien (IWT) in Bremen beschichtet. Während des Beschichtungsprozesses ergab sich eine maximale Prozesstemperatur von $\mathrm{T}_{\text {Prozess,max }}=140^{\circ} \mathrm{C}$. Beginnende Anlasseffekte für den Substratwerkstoff sind erst ab einer Anlasstemperatur von $\mathrm{T}_{\text {Anlass }}=160^{\circ} \mathrm{C}$ zu erwarten, sodass sich auch nachweißlich kein Verlust der Randhärte des einsatzgehärteten Substatwerkstoffs ergab [20]. Das Schichtsystem besteht, wie in Abb. 5 dargestellt, aus einer mit Titan angereicherten Festschmierstoffschicht $\mathrm{MoS}_{2}$ :Ti und einer TitanCarbon-Nitrid-Schicht (TiCN). Dabei dient die TiCN- 
Abb. 4 Zahnrad-Analogieprüfstand: Zwei-Scheiben-Tribometer

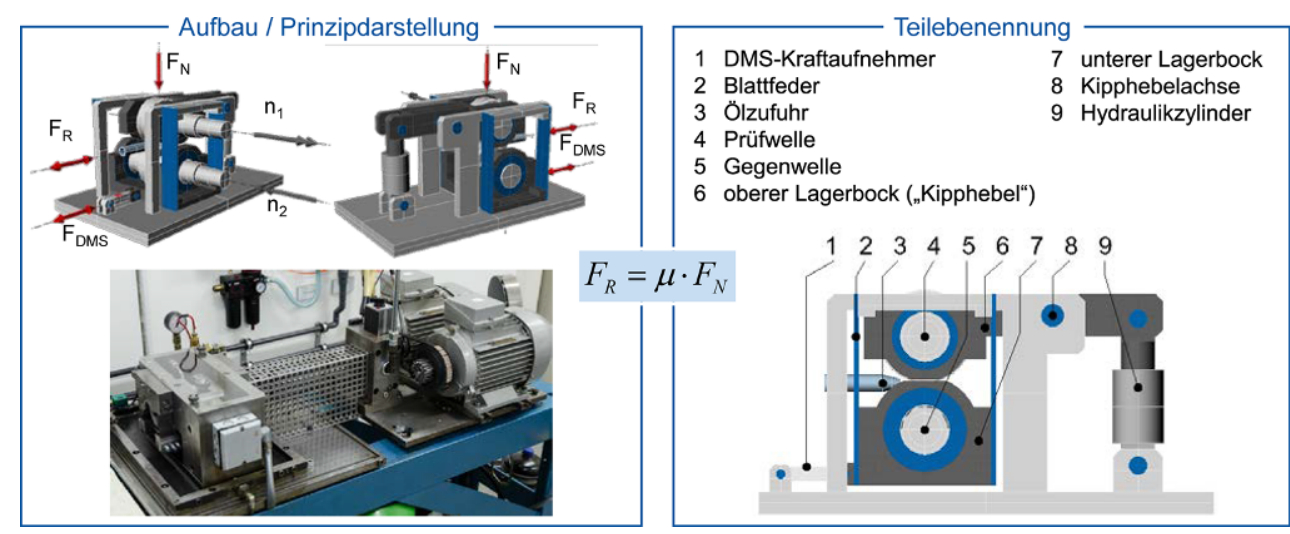

Abb. 5 Prüfkörper und Topographiemessung

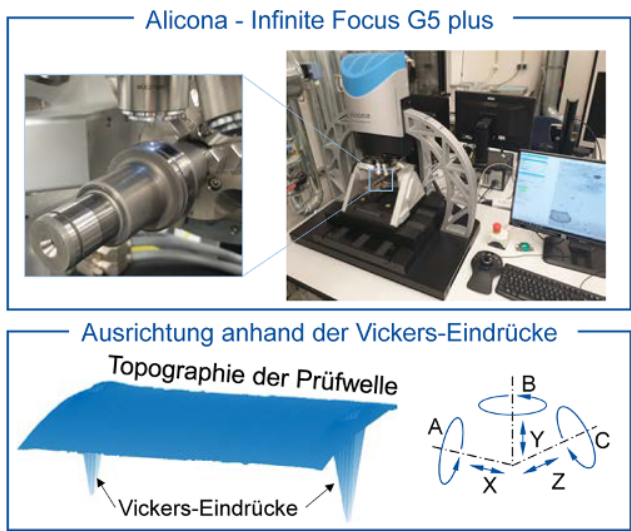

Schicht einerseits als Haftvermittler zwischen Festschmierstoffschicht und Substratwerkstoff. Andererseits hat die Zwischenschicht aufgrund ihrer hohen Festigkeit und dem hohen E-Modul eine Stützwirkung auf die Festschmierstoffschicht.

Das Schichtsystem wurde auf einen für die Zahnradherstellung üblichen Einsatzstahl $18 \mathrm{CrNiMo7}-6$ aufgebracht [20]. Mit der Einsatzhärtung wurde eine hohe Randhärte mit gleichzeitig zähem Kern erreicht. Durch die Folge aus Schleifen und Polieren wird eine Ausgangsrauigkeit von $\mathrm{Ra}=0,1-0,2 \mu \mathrm{m}$ mit einer Grenzwellenlänge von $\lambda c=0,08 \mathrm{~mm}$ erzielt. Die Gegenwelle ist auf dem Prüfabsatz mit einem $8 \mathrm{~mm}$ breiten Steg versehen, sodass im ZweiScheiben-Kontakt ein Linienkontakt vorliegt. Bei dem in diesen Untersuchungen zu erwartenden Schichtverschleiß bietet der Linienkontakt z. B. im Gegensatz zum Punktkontakt eine über den Prüflauf und die Kontaktbreite nahezu konstante Pressungsverteilung. Für die Verschleißanalyse der Prüfkörper auf Mikroebene wurden auf beiden Seiten neben der Laufbahn auf dem Prüfabsatz jeweils ein VickersEindruck erstellt. Daraufhin wurde die Topografie mit den Vickers-Eindrücken mit einem optischen 3D Messsystem, siehe Abb. 5, gemessen. Ein Algorithmus zur hochgenauen Ausrichtung mehrerer Topographiezustände ermöglicht eine präzise Verschleißauswertung [21].

\section{Laufzeituntersuchung im Zwei-Scheiben- Kontakt}

\subsection{Charakterisierung des Einsatzverhaltens}

Für den fluidfreien und mechanisch hochbelasteten Zahnkontakt wird das Einsatzverhalten eines $\mathrm{MoS}_{2}$ :Ti-TiCNSchichtsystems im Zwei-Scheiben-Kontakt untersucht. Zur Charakterisierung des Einsatzverhaltens wird das Reibkraftverhalten unter einer konstanten Linienpressung von $\mathrm{p}_{\max }=1648 \mathrm{~N} / \mathrm{mm}^{2}$ aufgezeichnet (Abb. 6). Die Versuche wurden mit einem Schlupf bezogen auf die Prüfwelle von $s_{\text {pruif }}=-20 \%$ und einer Summengeschwindigkeit von $\mathrm{v}_{\Sigma}=6,3 \mathrm{~m} / \mathrm{s}$ durchgeführt. Die Versuche wurden unter atmosphärischen Bedingungen und einer relativen Luftfeuchtigkeit von $30 \%$ durchgeführt.

In Abb. 6 sind die Reibkraft, der Reibkoeffizient und die Oberflächentemperatur über die Lebensdauer dargestellt. Die Reibkraftverläufe zeigen bei allen fünf Versuchen eine vergleichbare Charakteristik. Innerhalb der ersten Minute wird eine erhöhte Reibkraft beobachtet. Dieses Einlaufverhalten kann aufgrund der vorliegenden hohen Pressung mit einer Einglättung der Oberflächenrauheit in Verbindung stehen. Zudem können auch überlagerte Ausrichteeffekte der $\mathrm{MoS}_{2}$-Schicht im Einlauf zu einer erhöhten Reibkraft führen. Nach dem Einlauf weisen alle Versuche in einer 
Abb. 6 Reibverhalten im feststoffgeschmierten Wälzkontakt
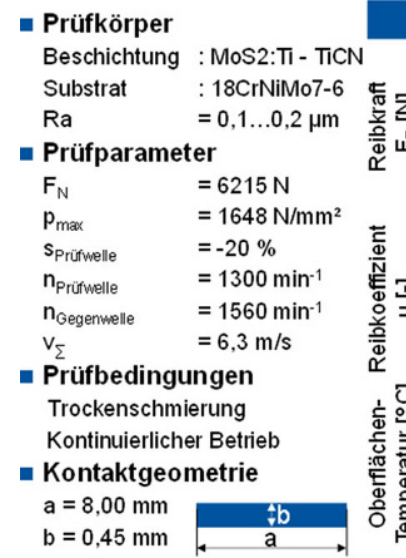

Einsatzverhalten: Reibkraft, Reibkoeffizient und Oberflächentemperatur

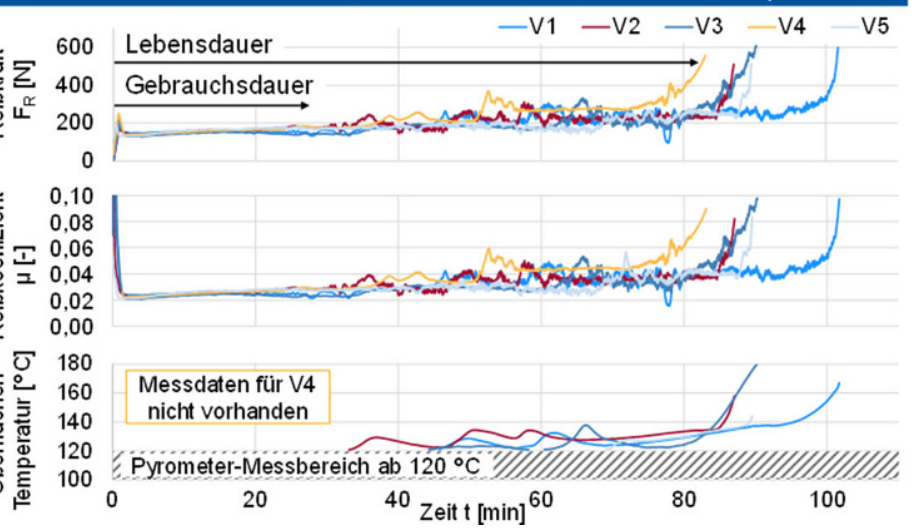

ersten Reibungsphase und analog zur Literatur einen stationären Reibkraftverlauf auf [15]. Die zweite Reibungsphase kennzeichnet sich durch eine instationäre Reibkraft und endet gleichzeitig mit der Lebensdauer durch einen exponentiellen Anstieg der Reibkraft. Der Reibkoeffizient liegt in der ersten Reibungsphase für alle fünf Versuche zwischen $\mu=0,02 \ldots 0,03$ und in der zweiten Reibungsphase zwischen $\mu=0,03 \ldots 0,06$. Eine mögliche Erklärung für den erhöhten Reibkoeffizienten in der zweiten Reibungsphase ist der lokale Verschleiß der Festschmierstoffschicht, sodass einzelne Bereiche oder Rauheitsspitzen ohne Festschmierstoff oder mit einer sehr geringen Schmierstoffschicht in den Kontakt kommen. Es wird vermutet, dass diese Bereiche in weiteren Kontaktwechseln wieder mit Festschmierstoff bedeckt werden und somit die Lebensdauer verlängern. Bei Versuchsabbruch werden Reibkoeffizienten von bis $\mathrm{zu} \mu=0,1$ aufgezeichnet. Der plötzliche starke Anstieg des Reibkoeffizienten könnte auf einen größeren Bereich mit verschlissener Festschmierstoffschicht zurückgeführt werden, sodass in diesem Bereich Adhäsion und Abrasion aufgrund des fehlenden Festschmierstoffs zu starkem Verschleiß führen. Diese Hypothese wird mit der Verschleißanalyse in Kap. 6 näher untersucht.

Aufgrund der fehlenden Kühlung eines flüssigen Schmierstoffs wurde bei den feststoffgeschmierten Versuchen die Oberflächentemperatur der Laufbahn auf der Prüfwelle gemessen. Die Temperatur wurde in Umfangsrichtung auf der gegenüberliegenden Seite von der Kontaktzone auf der Prüfwelle gemessen. Zur Temperaturmessung wird ein Zweifarben-Pyrometer (Sensortherm Metis M3) eingesetzt. Bei der Temperaturmessung wurden aufgrund des Messbereichs des Pyrometers nur Temperaturen oberhalb von $\mathrm{T}_{\min }=120^{\circ} \mathrm{C}$ aufgezeichnet. Des Weiteren beeinflussen die Oberflächenbeschaffenheit und der Verschleiß den Emissionskoeffizienten bei der Temperaturmessung, sodass die tatsächlichen Oberflächentemperaturen von der gemessenen Temperatur abweichen können. Die Temperaturverläufe in Abb. 6 zeigen, dass die Oberflächentempe- raturen bis kurz vor dem Versuchsabbruch unterhalb der maximalen Prozesstemperatur beim Beschichtungsprozess $\left(\mathrm{T}_{\text {Prozess } \max }=140^{\circ} \mathrm{C}\right.$, Kap. 4) bleiben. Jedoch werden in der zweiten Reibungsphase (d.h. im Bereich $\mathrm{t}=30-80 \mathrm{~min}$ ) Temperaturen zwischen $\mathrm{T}_{\text {Prüf }}=120-140^{\circ} \mathrm{C}$ beobachtet, sodass hier mögliche Verschleißmechanismen durch erhöhte Temperaturen der Prüfkörper begünstigt werden können. Aus der Literatur ist jedoch bekannt, dass lokale Temperaturen in der direkten Kontaktzone (Kontakttemperaturen oder Blitztemperaturen) im Festkörperkontakt weitaus höhere Werte erreichen [16]. Die Temperatur korreliert qualitativ und etwas zeitversetzt mit der Reibkraft, sodass die Temperaturmessung mit einem Pyrometer eine geeignete Messmethode für die Temperaturänderung während des Betriebs darstellt. Des Weiteren bildet die Kenntnis über die Korrelation zwischen Temperatur und Reibkraft eine Basis für ein Modell zur Vorhersage des Einsatzverhaltens mit Berücksichtigung der Temperatur.

\subsection{Einfluss von Intervallbetrieb auf den trockenen Wälzkontakt}

In Abb. 7 sind die Reibkraftverläufe von zwei weiteren Versuchen (V6 und V7) dargestellt. Bis auf die Unterbrechungen während der Prüfläufe wurden die Versuche unter identischen Prüfbedingungen, wie bei den zuvor gezeigten Prüfläufen (siehe Abb. 6), durchgeführt. Die Unterbrechungen wurden für die messtechnische Erfassung der Kontaktflächen genutzt, sodass die Prüfkörper wieder auf Raumtemperatur abgekühlt wurden. Das obere Diagramm zeigt den Reibkraftverlauf mit zwei Unterbrechungen im Prüflauf (Versuch V6). Die Unterbrechungen wurden dabei beide in der zweiten Reibungsphase des charakteristischen Einsatzverhaltens, bei etwa $t_{\mathrm{V} 6, \mathrm{U} 1}=61 \mathrm{~min}$ und $\mathrm{t}_{\mathrm{V} 6, \mathrm{U} 2}=76 \mathrm{~min}$, durchgeführt. Im Vergleich zu den Versuchen ohne Unterbrechung zeigt der Versuch ein vergleichbares Einsatzverhalten. Dies wird durch die Auswertung der Gebrauchs- und Lebensdauer in Abb. 7 bestätigt. Das Reibkraftverhalten der 
Abb. 7 Einsatzverhalten bei Intervallbetrieb
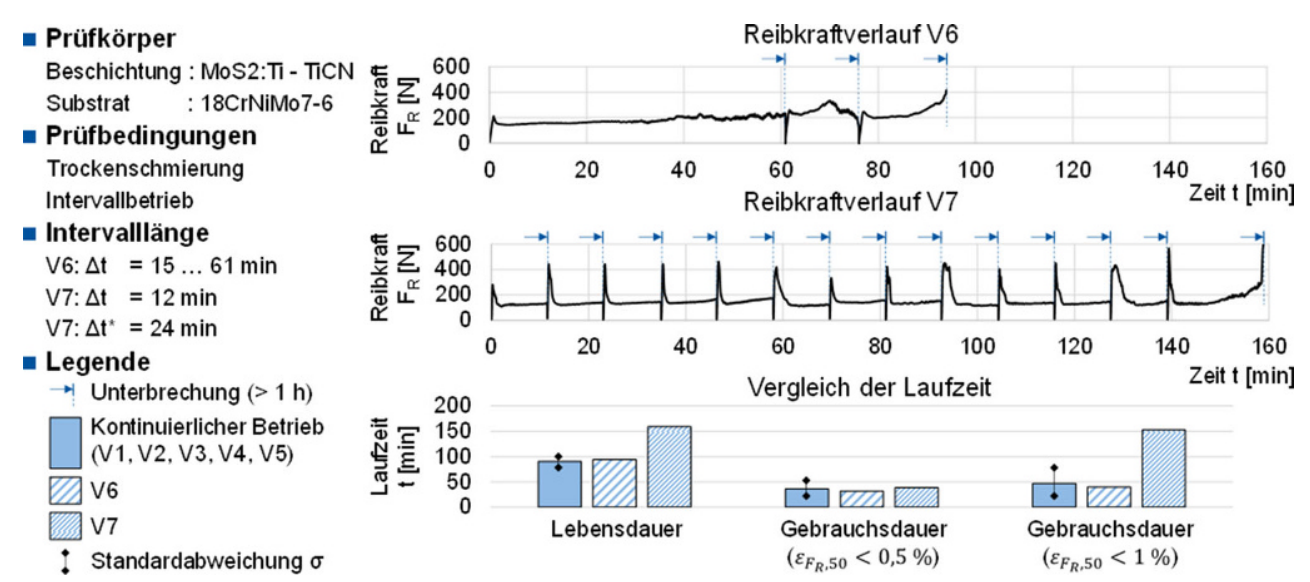

Vergleich der Laufzeit

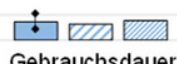

Gebrauchsdauer $\left(\varepsilon_{F_{R}, 50}<0,5 \%\right)$

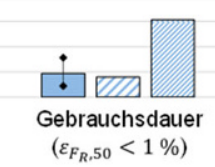

Versuche in Abb. 6 zeigt, dass der Übergang zwischen stationärer und instationärer Reibungsphase nicht sprunghaft ist. Für eine Quantifizierung der Gebrauchsdauer ist daher ein Kriterium zur Bestimmung des Übergangs notwendig. Dazu wurde zunächst der Reibkraftverlauf über einen Zeitraum von $\Delta t=50 \mathrm{~s}$ nach Gl. 1 geglättet, sodass nur signifikante Reibkraftänderungen berücksichtigt wurden. Die Gebrauchsdauer wurde dann durch eine relative Änderung der Reibkraft nach Gl. 2 definiert. Für die Versuche in dem vorliegenden Bericht wurde eine relative Reibkraftänderung nach Gl. 3 festgelegt. Der quantitative Vergleich des Reibkraftverhaltens und der qualitative Vergleich der Laufzeiten weisen darauf hin, dass Unterbrechungen in der zweiten Reibungsphase der Lebensdauer keinen maßgeblichen Einfluss auf das Einsatzverhalten des feststoffgeschmierten Wälzkontakts haben.

$$
\begin{aligned}
& F_{R, 50}(t)=\sum_{i=1}^{m=i+50} \frac{F_{R}\left(t_{i}\right)}{50} \\
& \varepsilon_{F_{R, 50}}=\frac{\left|F_{R, 50}\left(t_{i}\right)-F_{R, 50}\left(t_{i+1}\right)\right|}{F_{R, 50}\left(t_{i}\right)} \\
& \varepsilon_{F_{R, 50}}<\{0,5 ; 1\} \%
\end{aligned}
$$

\begin{tabular}{lclllll}
\hline $\mathrm{F}_{\mathrm{R}, 50}$ & {$[\mathrm{~N}]$} & $\begin{array}{l}\text { Geglättete } \\
\text { Reibkraft }\end{array}$ & $\mathrm{m}$ & {$[-]$} & $\begin{array}{l}\text { Anzahl } \\
\text { geglätteter } \\
\text { Werte }\end{array}$ \\
$\mathrm{i}$ & {$[-]$} & $\begin{array}{l}1,2,3 \ldots \\
\varepsilon_{F_{R, 50}}\end{array}$ & $\mathrm{t}_{\mathrm{i}}$ & {$[\mathrm{s}]$} & Zeit \\
& {$[\%]$} & $\begin{array}{l}\text { Relative } \\
\text { Reibkraftän- } \\
\text { derung }\end{array}$ & & & & \\
\hline
\end{tabular}

Das zweite Diagramm in Abb. 7 zeigt den Reibkraftverlauf des Versuchs V7 mit Intervallbetrieb in der ersten Reibungsphase, während der Gebrauchsdauer. Dabei wurde der Prüflauf jeweils nach einer Intervalldauer von $\Delta \mathrm{t}=12$ min unterbrochen. Die Lebensdauer des Wälzkontakts konnte somit deutlich gesteigert werden. Eine Hypothese zur Erklärung der verlängerten Lebensdauer ist, dass sich während der Versuchsunterbrechungen Oxidschichten auf den Kontaktflächen gebildet haben, die sich positiv auf die Beständigkeit der Festschmierstoffschicht auswirken [22]. Des Weiteren wurden erst kurz vor Versuchsabbruch bei $\mathrm{t}=158 \mathrm{~min}$ erhöhte Temperaturen mit $\mathrm{T}=138^{\circ} \mathrm{C}$ gemessen, sodass sich auch die geringere Temperatur der Prüfkörper positiv auf die Lebensdauer auswirken könnte. Im ersten Intervall ist der Reibkraftverlauf mit den vorherigen Versuchen vergleichbar (siehe Abb. 6). Auffällig sind jedoch die kurzzeitig stark erhöhten Reibkräfte nach jeder Unterbrechung. Dabei nimmt die Reibkraft in den Einlaufphasen in Bezug auf die initiale Einlaufphase tendenziell zu. Die initiale Einlaufphase lässt sich durch die Einglättungseffekte der Kontaktflächen, wie sie auch im ölgeschmierten Wälzkontakt beobachtet werden, erklären [23]. Eine mögliche Erklärung für die erhöhten Reibkräfte nach den Unterbrechungen könnte die Entstehung von Oxidschichten bzw. die Reaktion mit Sauerstoff $\mathrm{zu} \mathrm{MoO}_{3}$ geben. Diese $\mathrm{MoO}_{3}$ Verbindungen verbessern die Verschleißbeständigkeit und erhöhen den Reibkoeffizienten einer MoS2-Beschichtung [22]. Neben den Einlaufeffekten ist zu erkennen, dass der Wert der Reibkraft nach einem kurzen starken Anstieg immer wieder ein sehr niedriges Niveau anstrebt. Zudem weist die Reibkraft nach den Einlaufphasen einen nahezu stationären Zustand auf, wie er auch in der Gebrauchsphase der vorherigen Versuche zu sehen ist (siehe Abb. 6).

Aufgrund der langen Versuchsdauer bei V7 wurde nach 12 Intervallen die Intervalldauer auf $\Delta t^{*}=24$ min verdoppelt. In der Folge wurde der Versuch im Intervall 13 aufgrund einer stark ansteigenden Reibkraft beendet. Der quantitative Vergleich der Lebensdauer zeigt, das aufgrund der Unterbrechungen die Lebensdauer deutlich gesteigert werden kann. Auf Basis des Reibkraftverhaltens kann vermutet werden, dass die Lebensdauer ohne eine Verdopplung der Intervalldauer noch weiter gesteigert werden könnte. Dabei zeigt sich, dass die Gebrauchsdauer mit den Ergebnissen der vorherigen Versuche quantitativ vergleichbar ist. Bei der Auswertung der Gebrauchsdauer mit einer höheren zulässigen Änderung der Reibkraft von $\varepsilon_{\mathrm{FR}, 50}<1 \%$ 
Abb. 8 Verschleißanalyse im Intervallbetrieb (V6)
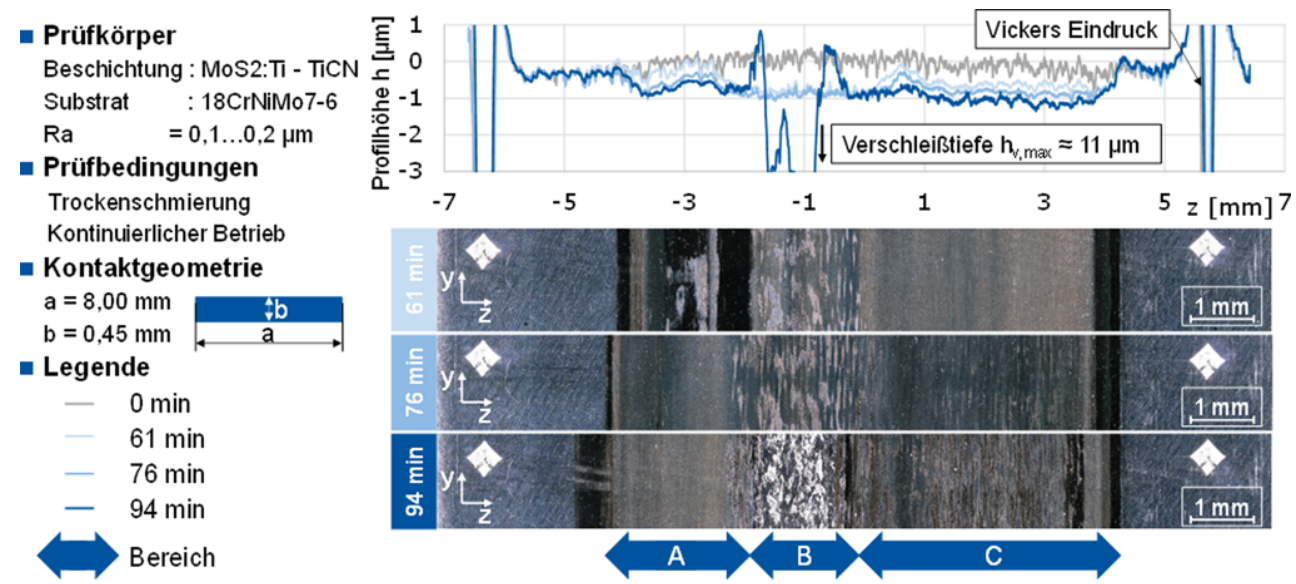

erreicht die Gebrauchsdauer für den Versuch V7 nahezu das Niveau der Lebensdauer. Während die Gebrauchsdauer der vorherigen Versuche gegenüber dem Kriterium von $\varepsilon_{\mathrm{FR}, 50}<0,5 \%$ nur wenig zunimmt (siehe Abb. 7). Neben der Reibkraftänderung kann als weiterer Aspekt auch das Niveau der Reibkraft für die unterschiedlichen Phasen der Lebensdauer verglichen werden. Bei dem Versuch V7 bleibt die Reibkraft abgesehen von den Einlaufphasen unterhalb von $F_{R}=200 N$. Während die Reibkraft der vorherigen Versuche nach der Gebrauchsdauer ein mittleres Niveau über $F_{R}=200 \mathrm{~N}$ annimmt. Somit kann festgehalten werden, dass für einen quantitativen Vergleich der Gebrauchsdauer zwischen Versuchen mit abweichendem Lastkollektiv sowohl die Reibkraftänderung als auch das gemittelte Niveau der Reibkraft betrachtet werden muss.

\section{Verschleißanalyse im feststoffgeschmierten Wälzkontakt}

Für eine detaillierte Analyse der Verschleiß- und Transfermechanismen im feststoffgeschmierten Wälzkontakt wurden die Unterbrechungen der Prüfläufe im Intervallbetrieb für die messtechnische Erfassung der Kontaktflächen genutzt. In Abb. 8 sind Messungen der Prüfwelle für den Versuch V6 mit zwei Unterbrechungen abgebildet. In dem Diagramm ist jeweils das Profil für die Zustände $\mathrm{t}=\{0 \mathrm{~min} ; 61 \mathrm{~min} ; 76 \mathrm{~min} ; 94 \mathrm{~min}\}$ der Prüfwelle dargestellt. Das Profil nach dem Ende bei $t=94 \mathrm{~min}$ zeigt einen starken Verschleiß mit einer Verschleißtiefe von $\mathrm{h}_{\mathrm{v}, \max } \approx 11 \mu \mathrm{m}$, sodass an dieser Stelle das Schichtsystem abgetragen wurde und der Substratwerkstoff frei liegt. Da der starke Verschleiß nicht im Randbereich der Laufbahn auftritt, kann ein Kantentragen im Tribometer ausgeschlossen werden. Bei dem direkten Vergleich der Profile ist zu erkennen, dass sich gegenüber dem Ausgangszustand $(\mathrm{t}=0 \mathrm{~min})$ eine bereits optisch klar erkennbare Laufbahn von ca. $b=8 \mathrm{~mm}$ entwickelt. Die Breite der Laufbahn entspricht der Breite des Steges der Gegenwelle. Des Weiteren ergibt sich aus dem Profilvergleich, dass die Verschleißtiefe über der Laufbahnbreite (Z-Richtung) nicht konstant ist. Für die folgende Verschleißauswertung wird die Laufbahnbreite daher in die Bereiche A, B und $\mathrm{C}$ eingeteilt, siehe Abb. 8. Im Bereich B ist der Verschleiß bei der ersten Unterbrechung bei $t=61$ min bereits stärker als im restlichen Bereich der Laufbahn. Dabei lässt eine Verschleißtiefe von $\mathrm{h}_{\mathrm{v}} \approx 1 \mu \mathrm{m}$ vermuten, dass ein Großteil der $\mathrm{MoS}_{2}: \mathrm{Ti}-$ Festschmierstoffschicht bereits verschlissen ist und in diesem Bereich bereits Teile der Hartstoffschicht aus TiCN freiliegend sind. Der Vergleich zu dem Profil von der zweiten Unterbrechung $(\mathrm{t}=74 \mathrm{~min})$ zeigt, dass in dem Bereich mit dem Schaden kein signifikanter Verschleiß mehr auftritt. Eine bereichsweise offenliegende TiCN-Schicht kann einen Zusammenhang mit der erhöhten Reibkraft in der zweiten Reibungsphase des Reibkraftverlaufs aufweisen.

Eine weitere Möglichkeit zur Analyse der Kontaktflächen bieten lichtmikroskopische Aufnahmen. In Abb. 8 ist jeweils der gleiche Ausschnitt von der Kontaktfläche auf der Prüfwelle für drei Zeitpunkte im Prüflauf abgebildet. Analog zu dem Profilvergleich sind auch auf den mikroskopischen Aufnahmen drei unterschiedliche Bereiche in der Laufbahn in Breitenrichtung zu erkennen. Bereich B kennzeichnet dabei den Bereich, in dem nach dem Versuch ein starker Verschleiß zu erkennen ist. Dieser Bereich weist bereits bei der ersten Unterbrechung eine inhomogene Oberfläche auf. Bei Bereich B ist zwischen $t=61 \mathrm{~min}$ und $\mathrm{t}=76 \mathrm{~min}$ die charakteristische Verteilung der helleren Anteile immer noch erkennbar. Aus dieser Entwicklung der Oberfläche kann geschlossen werden, dass in dem Bereich B die hellen Anteile im Prüflauf gewachsen sind. Aufgrund des starken Verschleißes und der fleckigen Optik liegt im Bereich B möglicherweise bereits die Hartstoffschicht TiCN frei. Dieser Umstand könnte die Zunahme der Reibkraft erklären, die in diesem Intervall detektiert 
wurde. Eine vergleichbare Entwicklung ist im Bereich $\mathrm{C}$ zwischen $t=76$ min und $t=94$ min zu erkennen. Jedoch ist der Verschleiß dort noch nicht so weit fortgeschritten wie im Bereich B. Im Bereich $\mathrm{C}$ bei $\mathrm{t}=61 \mathrm{~min}$ ist durch den Prüflauf eine relativ homogene Oberfläche entstanden, sodass sich der Verschleiß noch innerhalb der Schichtdicke der $\mathrm{MoS}_{2}$ :Ti-Beschichtung befindet. Diese Annahme wird durch den Profilvergleich bestätigt. Spezifische Verschleißmechanismen, wie beispielsweise ein Abblättern der Beschichtung, wurden auf den lichtmikroskopischen Aufnahmen nicht beobachtet werden. Die in Laufrichtung länglichen Muster auf der Laufbahn deuten auf einem abrasiven Verschleiß und Scherungseffekte im Schichtgittersystem hin.

\section{Zusammenfassung und Ausblick}

Der Stand der Technik zeigt, dass Festschmierstoffe eine geeignete Alternative für den hochbelasteten Zahnkontakt sind. Jedoch unterscheidet sich das Einsatzverhalten eines feststoffgeschmierten Wälzkontakts in Bezug auf Reibung und Verschleiß grundlegend von dem eines flüssiggeschmierten Wälzkontakts. Die Verschleißmechanismen und Einflussgrößen im feststoffgeschmierten Wälzkontakt sind noch nicht in dem Umfang erforscht, wie es für den flüssiggeschmierten Wälzkontakt der Fall ist.

Für den fluidfreien Einsatz im Zahnkontakt wurde ein Schichtsystem auf Basis von Molybdändisulfid im Zahnrad-Analogieversuch auf dem Zwei-Scheiben-Reibkrafttribometer untersucht. Das Schichtsystem besteht aus einer mit Titan angereicherten Festschmierstoffschicht $\mathrm{MoS}_{2}$ :Ti und einer darunter befindlichen TiCN-Hartstoffschicht. Die Reibkraft weist bei kontinuierlichem Betrieb und konstanter Kontaktpressung eine wiederholbare Charakteristik auf. Nach einer Einlaufphase bildet sich ein nahezu stationärer Zustand. Aufgrund von Schichtverschleiß entwickelt sich mit zunehmender Laufzeit eine instationäre Reibungsphase mit erhöhter Reibkraft bis zum Schichtversagen. Anhand der Charakteristik der Reibkraft kann zwischen einer Gebrauchs- und Lebensdauer unterschieden werden. Dabei zeigt die Lebensdauer eine bessere Wiederholgenauigkeit als die Gebrauchsdauer.

Für die Kenntnisse über die Verschleißmechanismen wurden in Bezug auf Gebrauchsdauer und Lebensdauer Versuche mit Intervallbetrieb durchgeführt und ausgewertet. Bei Unterbrechungen im Prüflauf während der instationären Reibungsphase ergab sich eine vergleichbare Gebrauchs- und Lebensdauer. Dagegen ergab sich durch Unterbrechungen in der stationären Reibungsphase eine erhebliche Steigerung der Gebrauchs- und Lebensdauer. Die Verschleißanalyse der Kontaktfläche auf der Prüfwelle zeigt, dass sich bereits zu Beginn der instationären Rei- bungsphase im Prüflauf ein starker Verschleiß in einem großen Teil der Laufbahn entwickelt hat.

Die abweichenden Kontaktbedingungen zwischen dem Zwei-Scheiben-Kontakt und dem realen Zahnflankenkontakt erfordern im nächsten Schritt eine entlang des Wälzwegs aufgelöste Untersuchung der Einlaufeffekte. Zur Überführung in die industrielle Anwendung sind die Untersuchungen auf die Fertigung, Beschichtung und auf den Betrieb von Prüfverzahnungen zu übertragen.

Förderung Gefördert durch die Deutsche Forschungsgemeinschaft (DFG) - 407625150.

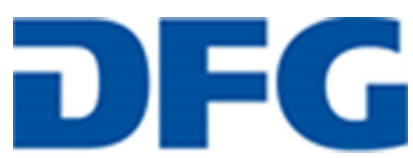

Funding Open Access funding enabled and organized by Projekt DEAL.

Interessenkonflikt S. Sklenak, J. Brimmers und C. Brecher geben an, dass kein Interessenkonflikt besteht.

Open Access Dieser Artikel wird unter der Creative Commons Namensnennung 4.0 International Lizenz veröffentlicht, welche die Nutzung, Vervielfältigung, Bearbeitung, Verbreitung und Wiedergabe in jeglichem Medium und Format erlaubt, sofern Sie den/die ursprünglichen Autor(en) und die Quelle ordnungsgemäß nennen, einen Link zur Creative Commons Lizenz beifügen und angeben, ob Änderungen vorgenommen wurden.

Die in diesem Artikel enthaltenen Bilder und sonstiges Drittmaterial unterliegen ebenfalls der genannten Creative Commons Lizenz, sofern sich aus der Abbildungslegende nichts anderes ergibt. Sofern das betreffende Material nicht unter der genannten Creative Commons Lizenz steht und die betreffende Handlung nicht nach gesetzlichen Vorschriften erlaubt ist, ist für die oben aufgeführten Weiterverwendungen des Materials die Einwilligung des jeweiligen Rechteinhabers einzuholen.

Weitere Details zur Lizenz entnehmen Sie bitte der Lizenzinformation auf http://creativecommons.org/licenses/by/4.0/deed.de.

\section{Literatur}

1. Sommer K, Heinz R, Schöfer J (2010) Verschleiß metallischer Werkstoffe. Erscheinungsformen sicher beurteilen, 1. Aufl. Praxis Werkzeugtechnik. Vieweg + Teubner, Wiesbaden

2. Niemann G, Winter H (2003) Getriebe allgemein, Zahnradgetriebe - Grundlagen, Stirnradgetriebe, 2. Aufl. Maschinenelemente, Bd. 2. Springer, Berlin, Heidelberg, New York

3. Hantschack F (2012) Trockenlaufende Stirnräder in der Raumfahrt. FVA (Forschungsvereinigung Antriebstechnik e.V.), Würzburg

4. Europäisches Parlament (2006) Maschinenrichtlinie 2006/42/EG

5. Isaacson AC, Wagner ME (2019) Oil-off characterization method using in-situ friction measurement for gears operating under lossof-lubrication conditions. Geartechnology 2019:46-54

6. Höhn B-R, Michaelis K (Hrsg) (2013) Low Friction Powertrain. Tragfähigkeit Low Loss Verzahnung. FVA (Forschungsvereinigung Antriebstechnik e. V.), Frankfurt a.M.

7. Höhn B-R, Grossl A, Diesselberg M, Martens S (2005) Development of powertrain and drive line components without liquid lubri- 
cation. Abschlussbericht. Technische Universität München, München

8. Schultz (1986) Nitrierte Stahlzahnräder trotzen dem Verschleiß. VDI Nachrichten 18:20

9. Krieger H, Günther D, Römhild I (1999) Studie zur Untersuchnung von Minimalmengenschmierung und Trockenlauf für Getriebe (Studie, Technische Universität München)

10. Miyoshi K (Hrsg) (2007) Solid lubricants and coatings for extreme environments. State-of-the-art survey. Glenn Research Center, Cleveland

11. Yilmaz M, Kratzer D, Lohnner T, Michaelis K, Stahl K (2018) A study on highly-loaded contacts under dry lubrication for gear applications. Tribol Int 128:410-420

12. Birkhofer H, Kümmerle T (2012) Feststoffgeschmierte Wälzlager. Einsatz, Grundlagen und Auslegung, 1. Aufl. Springer, Berlin, Heidelberg, New York

13. Gesellschaft für Tribologie (2002) Verschleiß, Reibung. Definitionen, Begriffe, Prüfung, Bd. 7. Gesellschaft für Tribologie, Aachen

14. ISO (2006) Calculation of load capacity of spur and helical gears. Basic principles, introduction and general influence factors (6336 Teil 1). ISO copyright office, Geneva

15. Kümmerle T (2012) Theorie und Evaluierung eines universall anwendbaren Gebrauchsdauermodells für feststoffgeschmierte Wälzlager. Konstruktionstechnik/Maschinenelemente, Bd. 415. VDI, Düsseldorf
16. Czichos H, Habig K-H (2010) Tribologie-Handbuch. Tribometrie, Tribomaterialien, Tribotechnik, 3. Aufl. Vieweg + Teubner, Wiesbaden

17. Singh H, Mutyala KC, Mohseni H, Scharf TW, Evans RD, Doll GL (2015) Tribological performance and coating characteristics of sputter-deposited Ti-doped MoS2 in rolling and sliding contact. Tribol Trans 58:767-777

18. Ping Z, Tianle W, Juanbo W, Min H, Yabo F, Yihang F, Feng S (2018) Study on synthesis and tribological properties characterization of MoS2-TiL/MoS2-TiH nano multilayer coating. Mater Sci Eng 423:12048

19. Löpenhaus C (2015) Untersuchung und Berechnung der Wälzfestigkeit im Scheiben- und Zahnflankenkontakt. Diss., RWTH Aachen University

20. Klocke F, Brecher C (2017) Zahnrad- und Getriebetechnik. Auslegung - Herstellung - Untersuchung - Simulation, 1. Aufl. Hanser, München

21. Brecher C, Löpenhaus C, Renkens D (2016) Vorhersagemodell für die geometrische Veränderung von Oberflächenstrukturen im Einlauf. In: WZL Getriebekreis (Hrsg) Arbeitstagung „Zahnrad- und Getriebeuntersuchungen"

22. Center PW (1988) Tribological performance of MoS2 compacts containing $\mathrm{MoO} 3, \mathrm{Sb} 2 \mathrm{O} 3$ or $\mathrm{MoO} 3$ and $\mathrm{Sb} 2 \mathrm{O} 3$. Wear 122(1):97-102

23. Lohner T, Mayer J, Michaelis K, Höhn B-R, Stahl K (2015) On the running-in behavior of lubricated line contacts. J Eng Tribol. https://doi.org/10.1177/1350650115574869 